\title{
El cáncer de la corrupción en Latinoamérica: El caso Odebrecht
}

\author{
The Cancer of Corruption in Latin America: The Odebrecht Case
}

\author{
Song Yuhui*
}

Resumen: En este artículo, tomando en cuenta el caso de corrupción de la constructora brasileña Odebrecht como ejemplo, se intentó determinar las causas y consecuencias de la influencia del caso de corrupción Odebrecht en la inestabilidad política de América Latina durante los últimos 15 años. A través de la revisión y el análisis de las bibliografías relacionadas se definen, hasta cierto punto, los factores teóricos y prácticos detrás de la corrupción política en los países de América Latina, especialmente en Brasil. Con el fin de encontrar un método relativamente factible, se proporcionan algunas sugerencias para el trabajo anticorrupción y la estabilidad política y social en la región, así como para la futura profundización académica.

Palabras clave: América Latina, corrupción política, Odebrecht, Brasil, estabilidad política, debilidad democrática.

Abstract: In this article, taking into account the corruption case of the Brazilian construction company Odebrecht as an example, I try to determine the causes and consequences of the influence of the Odebrecht corruption case on the political instability of Latin America during the last 15 years. Through the review and analysis of the related bibliographies, the theoretical and practical factors behind political corruption in Latin American countries, especially in Brazil, are defined to some extent. To find a relatively feasible method, some suggestions are provided for anti-corruption work and political and social stability in the region, as well as for future academic deepening.

Keywords: Latin America, political corruption, Odebrecht, Brazil, political stability, democratic weakness.

*Song Yuhui es profesor del Instituto Confucio en la Universidade de Cabo Verde, doctorando de la Faculdade de Ciências Sociais, Humanas e Artes de Uni-CV. Praça António Lereno, CP 379C, Praia, Santiago, Cabo Verde. Tels: +86 13017471556 y +238 590 0413. Correo-e: simao.song@qq.com. orCid: https://orcid.org/0000-0001-7760-795X.

Artículo recibido el 9 de julio de 2020 y aceptado para su publicación el 12 de marzo de 2021. 


\section{INTRODUCCIÓN}

T a corrupción en América Latina ha sido un problema sistemático a lo largo de su historia reciente, sobre todo en la última década, en que se tiene un foco claro de las proporciones de los males de la corrupción a través del caso representado por Odebrecht, que se expandió por toda la región. Por lo anterior, diversos gobiernos que aceptaron los sobornos de Odebrecht afectaron gravemente la legitimidad de las instituciones públicas y, en consecuencia, las democracias. La corrupción en su aspecto político presenta diferentes modalidades según la Organización de los Estados Americanos (OEA), como son enriquecimiento ilícito, nepotismo, tráfico de influencias, sobornos (oEA, 1998).

El caso de Odebrecht se caracteriza particularmente por ser un caso de soborno en escala masiva que ha afectado gravemente a los gobiernos latinoamericanos. Como ejemplos emblemáticos se observa la caída de presidentes como, por ejemplo, Ollanta Humala de Perú y Luiz Inácio Lula da Silva de Brasil, quienes enfrentaron juicio y han sido encarcelados debido a hechos relacionados con casos de corrupción impulsados por la constructora brasileña Odebrecht. Además, Perú ha experimentado otras secuelas de la corrupción relacionada con el caso en cuestión, con lo que el ex presidente Pedro Pablo Kuczynski, se vio obligado a dimitir en marzo de 2018 tras un escándalo de compra de votos. Este hecho supone otro escenario de debilidad política en la región que involucra a los actores políticos más relevantes.

El caso Odebrecht ha funcionado como un epicentro del cual se generó una compleja red de sobornos a lo largo de la región, cuyo centro se ubica en Brasil. Este país tiene especial importancia para América Latina, ya que se había mantenido como una de las economías más relevantes de la región; sin embargo, fue precisamente desde Brasil que se emitieron cuantiosos y numerosos hechos de sobornos por un tiempo considerablemente prolongado. El caso Odebrecht no solo ha afectado a figuras presidenciales, sino que además ha expuesto a una gran cantidad de funcionarios públicos que se encuentran implicados en el caso a lo largo de la región y, con ellos, han quedado evidenciadas unas instituciones públicas incompetentes.

Los constantes escándalos que surgieron una vez que se destapó el caso Odebrecht se perfilan en la preocupación sobre la actuación de los funcionarios latinoamericanos frente a procesos de sobornos que surgen de transacciones comerciales internacionales y que terminan enfermando a las instituciones democráticas, ya que impiden el progreso político, económico y social de la región, al mismo tiempo que dañan la imagen de las transnacionales latinoamericanas. 
Una de las consecuencias más contundentes es que la compañía Odebrecht se vio obligada a pagar la multa más alta de la historia en un escándalo de corrupción, mientras Brasil y los países afectados sufren las crisis políticas más agudas de la región. Especialmente para Brasil como centro de actuación de Odebrecht, la situación ha desencadenado la destitución de la presidenta Dilma Rousseff y la fragmentación de la cúpula política brasileña.

El ex presidente brasileño, Michel Temer también se ha visto envuelto en escándalos de corrupción, sufriendo la peor popularidad entre el pueblo brasileño. Debido a estos escándalos, la política brasileña que hasta hace poco gozaba de prestigio económico en el continente y que ha podido celebrar de manera casi consecutiva dos eventos de importancia internacional como lo son un Mundial de Futbol (2014) y unos Juegos Olímpicos (2016) se encuentra en estos momentos en una fase de recuperación frágil en la medida en que se siguen destapando casos de corrupción.

Brasil es considerado la potencia económica de latinoamericana, tanto por su extensión geográfica, como por los recursos económicos primarios con los que cuenta. Por ello, la crisis económica, política y social que ha desatado este caso de corrupción ha sido determinante para transformar la configuración política regional. De manera que esta investigación pretende precisar hasta qué punto Brasil con el caso Odebrecht ha sido decisivo en la evolución política de esta región.

Durante 2016 la palabra corrupción tuvo un lugar significativo en los análisis políticos y económicos sobre la región, destacando el caso Odebrecht como principal incidente en la elaboración del ranking del índice de percepción de la corrupción (IPC) latinoamericano. En la gráfica 1 se presentan los resultados del IPC con los sobornos realizados por la empresa. Venezuela resultó la nación más corrupta con un pago de soborno de 98 millones de dólares.

Curiosamente, aun con la crisis política brasileña vivida entre 2015 y 2016, y con la enorme cantidad de pagos en sobornos, se le percibe como la menos corrupta dentro de este ranking, y si se le ubica en la gráfica 1 se encuentra en quinto lugar como la menos corrupta de la región. Es un dato que hay que tener en cuenta dentro de los cambios políticos que ha sufrido Brasil y en la incorporación del presidente Temer dentro de esta dinámica.

\section{Antecedentes}

Tanto el caso de Petrobras como el de Odebrecht forman parte de la investigación Lava Jato (Lavado de autos), considerada la operación anticorrupción más grande de Brasil. Incluye la participación de un número importante de políticos, 
GRÁFICO 1. Índice de percepción de la corrupción en relación

\section{MÉXICO, ENTRE LOS MÁS CORRUPTOS DE AMÉRICA LATINA \\ Entre los países latinoamericanos donde la brasileña Odebrecht aceptó haber pagado sobornos a autoridades para conseguir contratos públicos, México tiene la tercera peor reputación en corrupción según el ránking 2016 de Transparencia Internacional.}

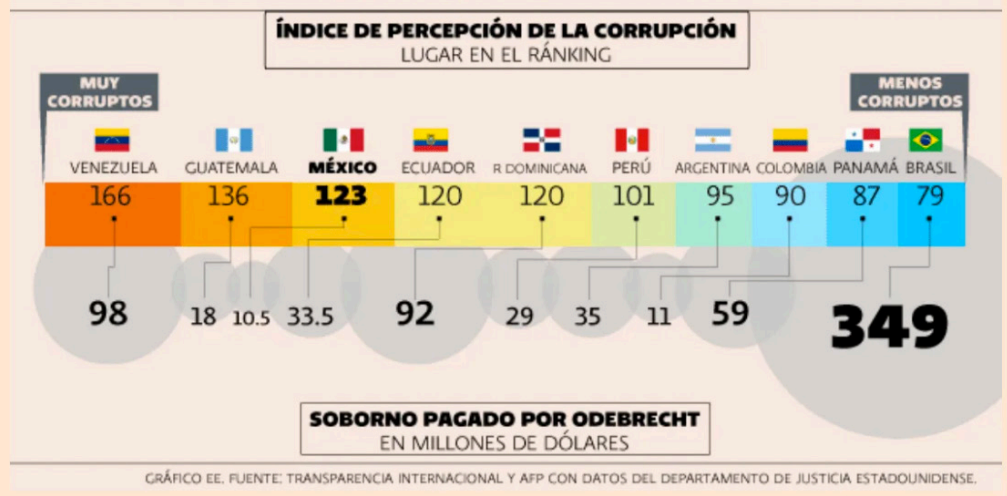

Fuente: Riquelme (2017).

funcionarios y empresarios que forman parte de una red fraudulenta gestada en la petrolera Petrobras.

La Operación Lava Jato inició en 2014 por decisión de la Corte Suprema Brasileña que decidió investigar a decenas de funcionarios con ciertos privilegios. Esta operación evidenció el caso de desviación de recursos, clientelismo y tráfico de influencias que sufre Brasil y varios de los países afectados por el caso Odebrecht. La operación comenzó en julio de 2013 cuando la Policía Federal de Curibita descubrió un caso de lavado de activos que relacionaba al experto en blanqueo de dinero Alberto Yousseff (Tible, 2015). Su testimonio junto con el de Paulo Roberto Costa, ex director de Abastecimiento de Petrobras, fue el inicio del descubrimiento de una operación de inmensas proporciones.

Petrobras licitaba sus obras con grandes empresas de ingeniería civil o construcción que estaban dentro del programa impulsado tanto por da Silva como por Rousseff para la creación de empleo. Para el beneficio de los contratos Petrobras pedía sobornos de 3 por ciento de los presupuestos que luego era distribuido entre diferentes políticos y demás empresarios. Para limpiar el dinero era invertido en hoteles, lavanderías y estacionamientos de gasolinas, para luego ser transferido hacia China a través de empresas fantasmas. La estrategia se repitió 
en diferentes países de América Latina (RPP Noticias, 2017a; 2017b) afectando en diferentes niveles los sistemas políticos de la región.

Odebrecht es una de las principales empresas brasileñas que está implicada en el caso. Se trata de la constructora más grande de Latinoamérica, por lo que los casos de corrupción no sólo se limitan a hacer negocios dudosos en Brasil, sino que también llevó la celebración de contratos fraudulentos en diferentes países de la región.

A continuación, el cuadro 1 presenta un estimado de las cifras que se produjeron para el soborno o pagos a funcionarios corruptos y partidos políticos en los países latinoamericanos. Se puede observar como Brasil destaca con una enorme cifra que casi alcanza los 350 millones de dólares, seguida por Venezuela que casi llega a los 100 millones de dólares y que en general se producen en los principales países y mercados comerciales de América Latina como República Dominicana, Panamá, Argentina, Ecuador, Perú, Guatemala, Colombia y México.

Aunque no puede afirmarse que la actuación de Odebrecht es la principal responsable de la crisis y los desequilibrios políticos y económicos que se registran en los países señalados, sí es un reflejo de que la legitimidad de sus gobiernos e instituciones tiene una deuda con sus ciudadanos. Si se compara el índice de percepción de la corrupción con el cuadro 1 se evidencia por ejemplo que en el caso de Venezuela, que fue el segundo país con una mayor cantidad de soborno,

CUADRO 1. Pagos corruptos a funcionarios extranjeros y partidos políticos en los países

\begin{tabular}{lc}
\hline País & Sobornos realizados \\
\hline Brasil & 349.0 \\
Venezuela & 98.0 \\
República Dominicana & 92.0 \\
Panamá & 59.0 \\
Argentina & 35.0 \\
Ecuador & 33.5 \\
Perú & 29.0 \\
Guatemala & 18.0 \\
Colombia & 11.0 \\
México & 10.5
\end{tabular}

Fuente: Departamento de Justicia de Estados Unidos (2016a). 
hoy en día la corrupción de su gobierno y sus instituciones resulta la más grave del continente. Lo que Odebrecht viene a destapar es que los países en los cuales no existe una democracia fuerte, su grado de actuación es mucho más accesible, mientras que la estabilidad política del país es mucho más frágil.

En el extremo contrario se encuentra Brasil donde se encuentran implicados los ex presidentes Lula da Silva y Dilma Rousseff, quienes actualmente enfrentan acusaciones y apertura de expediente de investigación; una vez que se toman las medidas correctivas como estas averiguaciones, la percepción sobre la corrupción cambia a pesar de que fue en Brasil donde se originó la red de sobornos impulsada por Odebrecht.

\section{Objetivo de estudio}

Objetivo general:

- Determinar las causas y consecuencias de la influencia del caso de corrupción Odebrecht en la inestabilidad política de América Latina durante los últimos 15 años.

Objetivos específicos:

- Definir las actuaciones de la empresa constructora brasileña Odebrecht en los escándalos de corrupción de América Latina, con el fin de determinar la influencia que tuvo en el devenir político de la región.

- Establecer las acciones y consecuencias de los sobornos realizados por Odebrecht en cada uno de los países afectados.

- Señalar el alcance de la crisis política generada en Brasil a partir de los escándalos de corrupción originados por la constructora Odebrecht.

\section{Justificación}

Existe una percepción generalizada de que América Latina experimenta consecutivamente problemas de corrupción como parte de su idiosincrasia histórica. Especialmente, en los últimos 15 ańos ha estallado una serie de casos de corrupción que no han sido aislados, sino que parten de una misma red corruptiva que tiene como epicentro Brasil y particularmente la constructora Odebrecht.

Cabe preguntarse entonces si esta "tradición" de caer constantemente en situaciones de corrupción política ha llegado a un nivel de consolidación que ha logrado establecer un sistema corruptivo por un periodo que supera la década y que además tenga un alcance regional.

El caso Odebrecht ha llamado la atención del mundo por estas características y porque también ha afectado a una variedad de líderes políticos con tendencias 
GRÁFICA 2. Crecimiento del PIB (variación anual) (porcentaje)
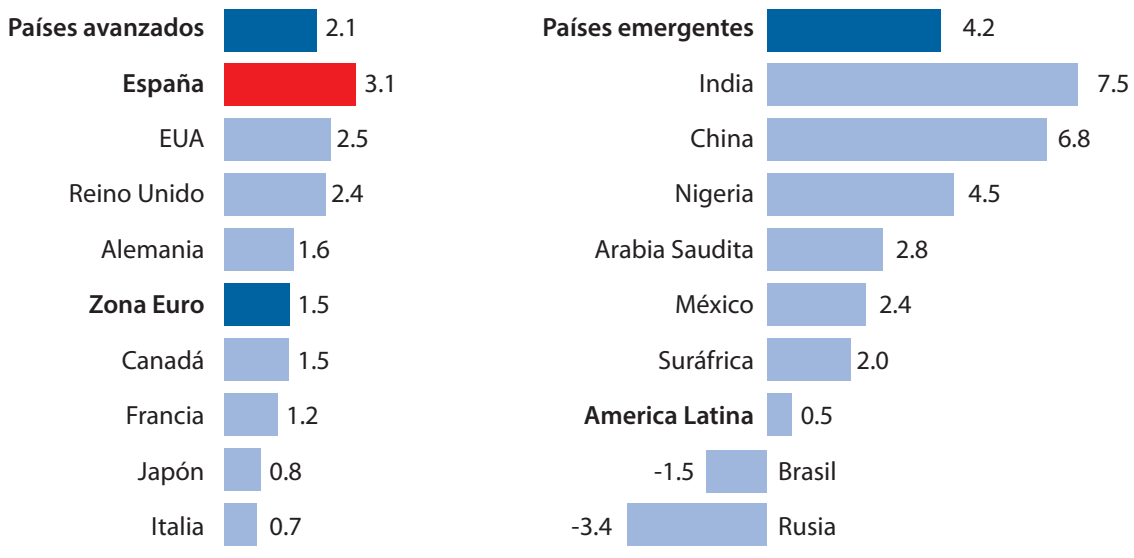

Fuente: Fondo Monetario Internacional (2015).

políticas muy diferentes. En este sentido, la corrupción política impulsada por esta empresa cubre un amplio espectro político como lo es el populismo de izquierda de Lula da Silva, o de tendencia de centro-derecha como lo es el caso de Kuczynski.

En este sentido, la intervención de Odebrecht en sobornos para facilitar el desarrollo de grandes proyectos, coincide con la perpetuación de líderes políticos que actualmente llevan expedientes judiciales relacionados con Odebrecht. Esto evidencia actos de soborno y clientelismo. Por ello esta investigación pretende aportar el análisis sobre las causas y consecuencias que ha tenido en la región centrándose en la debilidad de los sistemas políticos reflejada en la legitimidad, la estabilidad de los sistemas políticos y la eficacia institucional.

Esta investigación servirá como base para profundizar sobre una de las causas por las cuales la región de América Latina presenta un crecimiento de su desarrollo menor del que se espera, y se mantenga con cierto retraso respecto a otras regiones con mayor desarrollo. La debilidad de las democracias latinoamericanas ha incidido en su crecimiento y desarrollo, por lo que acciones como la de Odebrecht pueden considerarse de gran amenaza para el crecimiento de la región. En la gráfica 2 se muestra la comparación entre los países desarrollados y los emergentes, en los que la región se ve bastante desfavorecida

Por lo anterior, esta investigación pretende ser un aporte a la evaluación sobre la actuación de Odebrecht en el estado de inestabilidad política que afecta a la región, según los siguientes lineamientos. 


\section{METODOLOGÍA Y FUENTES}

\section{Método de investigación}

Es un trabajo de investigación bibliográfica en la cual se procede a la revisión exhaustiva de fuentes tanto oficiales como periodísticas y especializadas, con el fin de determinar las causas y consecuencias del caso de corrupción de Odebrecht en América Latina dentro del espectro político.

Se trata de un análisis de amplio alcance geográfico, es decir, de proporción regional, en el que se toma en cuenta el contexto político basada en actos corruptivos a lo largo de la región en los últimos 15 años. Los estudios regionales tienen por objetivo analizar cómo se ha articulado un fenómeno de manera integral y que evidencia las tensiones entre lo local y lo global en los diferentes ámbitos de lo económico, lo político, lo histórico y lo cultural.

El rasgo esencial de la regionalización se cimienta en un ejercicio delimitativo centrado en cuestiones geográficas, culturales, perceptivas, políticas o económicas que se entrecruzan o definen a partir del juego individual y colectivo de lo social (Rózga-Luter y HernándezDiego, 2010).

Por lo tanto, se tiene un espacio de análisis que abarca América Latina, en un periodo que abarca los últimos 15 años, y un problema de estudio que es establecer las causas y consecuencias de la corrupción en el devenir político latinoamericano. El hilo conductor será el de los efectos de la corrupción impulsada por Odebrecht en el estado político de la región.

\section{Fuentes}

En cuanto a las fuentes secundarias se dispone de artículos periodísticos y artículos especializados en politología y economía, trabajos monográficos sobre el estado generalizado de la corrupción en América Latina. Por parte de las fuentes primarias se tomarán en cuenta informes de instituciones internacionales como el Banco de Desarrollo de América Latina (CAF), la OEA y La Comisión Económica para América Latina (CEPAL). Estudios políticos sobre la última década en América Latina y fuentes emitidas por los países afectados.

\section{Problema de la investigación}

En la actualidad el problema de la corrupción se considera uno de los aspectos de gobierno que frena el desarrollo de los países que se encuentran en transición hacia países de primer mundo. En este sentido, los escándalos de corrupción provocados por la transnacional brasileña Odebrecht en complicidad con los diferentes 
líderes de gobierno latinoamericanos durante los últimos 15 años, ha representado el atraso económico de ciertos países como Venezuela, Argentina, y la crisis política que Brasil está atravesando, entre otros.

No es posible afirmar que la actuación de Odebrecht es la única causa del proceder corruptivo de la región, pero sí en definitiva que la red corruptiva que ha formado a lo largo de América Latina así como el largo periodo de su actuación han influido de manera bastante significativa.

Tomando esto en cuenta se establecen las siguientes cuestiones de investigación que orientarán el desarrollo y análisis de la misma:

1. ¿Cómo se relaciona el caso de Odebrecht con el estado de inestabilidad política que ha sufrido la región latinoamericana durante los últimos 15 ańos?

2. ¿En qué medida ha afectado la legitimidad de las democracias de los países afectados?

3. ¿Cómo se han visto afectadas la eficacia y la legitimidad de las instituciones públicas a partir de la intervención de Odebrecht en actos de corrupción?

4. ¿En qué medida Brasil ha funcionado como epicentro de donde se despliega la red corruptiva iniciada a partir del caso Odebrecht?

\section{ESTADO DE LA CUESTIÓN}

En una revisión previa de las fuentes se tiene que los artículos periodísticos y artículos especializados en politología y economía son los más abundantes. Por otro lado, aunque existen al menos diez países afectados, no todos ellos han recibido la misma atención internacional.

Aun así, es posible conseguir información de todos ellos, de manera que se podrán contrastar la diversidad de fuentes diferentes. Por otro lado, existen trabajos sobre el estado general de corrupción en América Latina, por lo que habría que profundizar en lo particular del caso Odebrecht debido al hecho de que por un tiempo ha podido tener una influencia considerable sobre el destino político reciente de la región.

Entre las fuentes consultadas para lograr una definición y delimitación cabal del tema se tiene la del autor Manuel Villoria Mendieta (2006), "¿Por qué desconfiamos de los políticos? Una teoría sobre la corrupción de la política"; este artículo se centra en seńalar los mecanismos que legitiman el liderazgo público democrático durante el siglo xxI, haciendo énfasis en la conciencia del deterioro de las relaciones entre los políticos y la ciudadanía como consecuencia de la corrupción. 
Otros aspectos que se toman en cuenta en este trabajo es el abordaje de la ética como problema de fondo de la corrupción, además de ser definitiva en la ruptura del contrato moral que adquiere el personaje líder con la ciudadanía. Por último, este artículo expone reflexiones sobre las diferencias entre países desarrollados, como en vías de desarrollo respecto al deterioro de las democracias.

Otro trabajo revisado es el de Mansilla (2000), "Algunas insuficiencias de la democracia contemporánea (una crítica de las teorías de la transición latinoamericana)". Este artículo realiza un análisis sobre los países considerados del Tercer Mundo en el cual se presentan modelos altamente centralizados que contribuyen a la burocratización de la administración pública, y por lo tanto da pie a situaciones en las cuales se da espacio a la corrupción.

Sobre todo, el artículo expone cómo las sociedades a las cuales se les somete a experimentos socialistas no terminan siendo sociedades más justas.

Además el autoritarismo conlleva a que cada vez haya menos oportunidad de la renovación de los poderes públicos, facilitando la corrupción a gran escala. Por último, se debate el tema de que en América Latina la propensión a la corrupción proveniente del clientelismo y de la mediocridad de los gobernantes quizá resulte exagerada.

En el artículo escrito por el autor Covarrubias González (2005), "La globalización de la corrupción: Un efecto perverso de las transformaciones recientes en la política y la democracia”, se plantean las principales direcciones que ha tomado la corrupción como flagelo político-social asociado a la democracia. El autor plantea que, aunque existe una aparente relación entre corrupción y democracia, no existen suficientes trabajos que exploren este fenómeno.

Además, el autor señala que no se ha realizado una historia conceptual sobre la corrupción desarrollada dentro del ámbito de estados democráticos, en particular después de la Segunda Guerra Mundial. Es por ello que el artículo aborda el flagelo de la corrupción registrado en la última década del siglo xx.

Incluye la importancia de los medios de comunicación, así como la opinión pública, en los escenarios tanto nacionales como internacionales. Sobre todo, la corrupción en los últimos tiempos ha tenido una importancia relevante en las dinámicas internacionales.

El texto elaborado por Patińo Aristizábal (2007), "El neopopulismo en el contexto de la democracia latinoamericana", realiza un estudio sobre el populismo político en el contexto latinoamericano, señalando los rasgos que le han permitido adaptarse a los inicios del siglo xxi. Este artículo tiene su importancia en el cuestionamiento de si el populismo ha resultado un rasgo característico de la región. 
Respecto a la corrupción, relaciona la aparición de personajes mesiánicos como la figura del fallecido presidente venezolano Hugo Chávez, quien ha contribuido a transformar las instituciones públicas en caldos de cultivo para la corrupción al tiempo que le ha permitido mantenerse en el poder de forma sostenida.

Pero, más allá, la relación entre corrupción y líder populista está presentada en este trabajo a través de la promesa de estos políticos en su lucha contra de la corrupción, como una especie de enemigo común público que hay que combatir, y que forma parte del discurso populista.

Por último, mencionaremos el trabajo del autor Rodríguez Kauth (2011), "Corrupción e impunidad: Dos estilos de cultura política latinoamericana". Este artículo contiene una revisión histórica de la corrupción en América Latina con énfasis en el caso argentino. Incluye las posibles definiciones de corrupción e impunidad, y los posibles escenarios en los que se pueda establecer la relación de ambos conceptos.

Asimismo, este trabajo incluye las implicaciones éticas de la práctica de la corrupción y la impunidad y el deber que los académicos tienen al respecto al plantearse una perspectiva valorativa. El artículo concluye con el señalamiento de las acciones ciudadanas como un elemento de importancia para acabar con la práctica de ambos fenómenos.

Como trabajo especializado se presenta la obra de Quiroz (2014), Historia de la corrupción en el Perú, la cual ofrece una perspectiva histórica del siglo xx e inicios del xxi sobre la problemática de la corrupción en diferentes periodos, además de establecer las consecuencias políticas que ha provocado.

Otro trabajo especializado en corrupción dentro del ámbito nacional es la obra de Neely Ivanovic (2010), Crónica de la corrupción a la Chilena. Este trabajo habla en particular de las instituciones políticas chilenas al servicio de la corrupción y de empresas constituidas por el Estado chileno para fines ilícitos. La obra se encuentra separada por periodos históricos del siglo XX y XXI, de los cuales es preciso tomar el periodo que va del ańo 2000 a 2010.

Por último, entre los textos especializados se cita la obra de Westin (2017), La caída de Dilma, en el que se trabaja en profundidad sobre el impeachment que sufrió la ex presidenta a mediados de 2016.

En cuanto a instituciones internacionales es importante destacar los trabajos realizados por el CAF, los cuales han profundizado en el tema de la corrupción como problema prioritario en el desarrollo de América Latina. En este sentido, se citan los siguientes trabajos: 
El trabajo sobre corrupción incluido en la investigación Temas críticos para América Latina (2002) resalta para esta fecha que la corrupción es uno de los problemas más generalizados en la región presentes tanto en el ejercicio público como privado, que ha corrompido las instituciones democráticas en aquellos países que padecen este problema. Al respecto, señala una serie de consecuencias o efectos que tienen principal repercusión en las economías nacionales afectando a su vez, los niveles de crecimiento y vulneración de las crisis sociales locales.

Este trabajo menciona diferentes consecuencias principales que se dan a partir de la práctica de la corrupción en la región, a saber, distorsiones en la economía en la que se presentan contratos irregulares, contratos realizados a partir de sobornos, desviación de la actividad productiva y disminución del crecimiento. Así como clientelismo, e ineficiencia en la administración pública y privada.

$\mathrm{Al}$ mismo tiempo, este trabajo señala que la percepción de corrupción se ha visto incrementada, sobre todo en Venezuela que para 2002 se estima que los costos de corrupción alcanzan unos 517 millones de dólares sobre el patrimonio nacional.

Uno de los aspectos más importantes de este trabajo es que propone una línea de acción regional contra la corrupción; esto es, ahondar y aplicar en las propuestas de los organismos internacionales como la OEA, el Banco Interamericano de Desarrollo (BID), el Banco Mundial y la CEPAL. Al respecto publica una tabla que muestra las recomendaciones comunes de los tratados internacionales para el combate de la corrupción. Se insiste en la cooperación internacional, y en la valoración de instituciones vigilantes y colaboradoras que son especialistas en la materia.

En la publicación de CAF, Focus América Latina núm. 7 (2015), se incluye el artículo que lleva por título "Cómo balancear entre autonomía y control en el manejo de las compras públicas", que es importante para este trabajo porque incluye medidas precisas de cómo disminuir los actos de corrupción en los entes públicos. La principal medida que propone este trabajo tiene que ver con el monitoreo directo de instituciones autónomas sobre las acciones administrativas de las instituciones públicas con el fin de disminuir los desvíos de capital.

Las tareas de monitorización incluyen las propuestas de compras públicas, investigación profunda sobre los proveedores, y evaluación de impacto sobre las compras o posibles proyectos sobre el bienestar general de la población al que están dirigidos. Por otro lado, este artículo coincide en que una de las principales consecuencias de la corrupción es el freno del desarrollo y la deslegitimación de las instituciones públicas, lo que afecta a las democracias nacionales. 
Respecto a trabajos realizados por la Organización de los Estados Americanos, se tiene como documento fundamental la Convención Interamericana Contra la Corrupción (1996), adoptado en la ciudad de Caracas, Venezuela, en el año 1996 durante la Conferencia Especializada sobre el Proyecto de Convención Interamericana Contra la Corrupción; entró en vigor en 1997 conforme al artículo XXV de la Convención Interamericana Contra la Corrupción.

En este tratado se encuentran suscritas 35 naciones incluyendo las analizadas en este trabajo. Al respecto nos referiremos en particular a los artículos I-3 y I-4 sobre el soborno transnacional y enriquecimiento ilícito, respectivamente:

3. Soborno transnacional. La legislación actual de Estados Unidos prevé sanciones penales para el soborno transnacional. Por lo tanto, Estados Unidos entiende que no se precisa de una legislación adicional para que ese país cumpla con la obligación impuesta en el artículo VIII de la Convención.

4. Enriquecimiento ilícito. Estados Unidos pretende brindar asistencia y cooperación a los otros Estados partes, de conformidad con el párrafo 3 del artículo IX de la Convención, hasta donde le permita su legislación interna. Estados Unidos reconoce la importancia de combatir el enriquecimiento indebido de los funcionarios públicos, y cuenta con leyes penales para impedir o castigar tal conducta. Estas leyes obligan a los funcionarios superiores del gobierno federal a presentar declaraciones financieras verdaderas, obligación que de no cumplirse está sujeta a sanciones penales. También permite el enjuiciamiento de los funcionarios públicos del gobierno federal que han evadido impuestos sobre riqueza obtenida ilícitamente (OEA, 1996).

La revisión de este tratado internacional establecido por el ente de mayor jerarquía en América es fundamental para el desarrollo de esta investigación debido a que es precisamente Estados Unidos quien abre el expediente en contra de la transnacional brasileña Odebrecht, y que además se compromete al intercambio de información con los demás Estados miembros para el esclarecimiento de las acciones corruptivas en los países que aquí analizamos.

Por otro lado, el documento es explícito en su Artículo VIII sobre el soborno transnacional, al respecto expone que cada Estado expresa:

[...] el acto de ofrecer u otorgar a un funcionario público de otro Estado, directa o indirectamente, por parte de sus nacionales, personas que tengan residencia habitual en su territorio y empresas domiciliadas en él, cualquier objeto de valor pecuniario u otros beneficios, 
como dádivas, favores, promesas o ventajas, a cambio de que dicho funcionario realice $\mathrm{u}$ omita cualquier acto, en el ejercicio de sus funciones públicas, relacionado con una transacción de naturaleza económica o comercial (OEA, 1996).

Como es evidente, la incorporación de este documento en el estudio del caso Odebrecht, debido a su naturaleza internacional, tiene la mayor pertinencia. Sobre los problemas que han desatado las acciones corruptivas de esta empresa en la región, es posible desarrollar la consolidación de las estructuras y redes de corrupción a lo largo de la región, la erosión de la credibilidad de los gobiernos, en particular de Brasil, Argentina, Ecuador y Venezuela, en los cuales no se ha superado la crisis política que les afecta.

En cuanto a documentos emitidos por la CEPAL se presenta el trabajo Corrupçáo e controles democráticos no Brasil (Avritzer y Filgueira, 2011), que profundiza sobre las medidas jurídicas, administrativas y de control público que Brasil ha emprendido a lo largo de la primera década del siglo xxi. Tiene su importancia en comprender los avances que ha hecho Brasil en la lucha contra la corrupción, los cuales han permitido en gran medida adelantar las investigaciones que se han realizado en el caso Odebrecht en la actualidad.

\section{ANÁLISIS DEL ALCANCE DE LA CORRUPCIÓN POLÍTICA IMPULSADA POR LA CONSTRUCTORA ODEBRECHT SOBRE LATINOAMÉRICA}

En el presente análisis se tomarán en cuenta la definición y las características de los actos de corrupción que han partido de la intervención de Odebrecht conceptualizando los criterios y características. Para una mayor comprensión del alcance del caso, en el cuadro 2 se presentan los pagos realizados por la constructora.

Además, el análisis toma en cuenta las tres variables establecidas en el marco metodológico y desarrollado en el marco teórico, como son la corrupción política en el contexto de legitimidad, estabilidad política e institucional atendiendo a sus causas y consecuencias.

En el cuadro 2 se exponen los sobornos efectuados por país. Este cuadro muestra también los beneficios aproximados que se obtuvieron en los países en los que se practicó el soborno y el concepto en el espacio correspondiente entre los respectivos ańos de la práctica de la corrupción.

\section{Características del caso Odebrecht}

En primer lugar el hecho de corrupción que define el caso de Odebrecht corresponde a una serie de sobornos conocidos como coimas que operaron en al menos doce países latinoamericanos con la finalidad de conseguir facilidades de contratos 
CUADRO 2. Pagos corruptos a funcionarios extranjeros y partidos políticos en los países

\begin{tabular}{|c|c|c|c|}
\hline Pais & $\begin{array}{l}\text { Soborno } \\
\text { (millones } \\
\text { de dólares) }\end{array}$ & Cómo ocurrió & $\begin{array}{l}\text { Beneficio } \\
\text { obtenido de } \\
\text { la operación } \\
\text { (dólares) }\end{array}$ \\
\hline Brasil & 349.0 & $\begin{array}{l}\text { Entre } 2003 \text { y 2016, Odebrecht pagó sobornos a partidos políticos, } \\
\text { funcionarios extranjeros y funcionarios públicos, con el fin de obtener } \\
\text { una ventaja en la adjudicación de proyectos. }\end{array}$ & 1.9 billones \\
\hline Venezuela & 98.0 & $\begin{array}{l}\text { Entre } 2006 \text { y } 2015 \text {, Odebrecht realizó pagos corruptos a funcionarios } \\
\text { gubernamentales e intermediarios que trabajan en su nombre, con el } \\
\text { fin de obtener y retener contratos de obras públicas. Los intermediarios } \\
\text { pagaban sobornos a los funcionarios del gobierno en nombre de la } \\
\text { compañía, para influir en la asignación de recursos a los proyectos y } \\
\text { obtener información confidencial sobre precios y ofertas. }\end{array}$ & $\begin{array}{l}\text { No } \\
\text { especificado }\end{array}$ \\
\hline $\begin{array}{l}\text { República } \\
\text { Dominicana }\end{array}$ & 92.0 & $\begin{array}{l}\text { Entre } 2001 \text { y 2014, Odebrecht realizó pagos corruptos a funcionarios } \\
\text { gubernamentales e intermediarios que trabajaban en su nombre en } \\
\text { la República Dominicana. A través de este acuerdo, Odebrecht pudo } \\
\text { influir en el presupuesto gubernamental y en las aprobaciones de } \\
\text { financiamiento para llevar a cabo proyectos en el país. }\end{array}$ & 163 millones \\
\hline Panamá & 59.0 & $\begin{array}{l}\text { Entre } 2010 \text { y } 2014 \text {, Odebrecht realizó pagos corruptos a funcionarios } \\
\text { gubernamentales e intermediarios para asegurar, entre otras cosas, } \\
\text { contratos de obras públicas. Entre } 2009 \text { y } 2012 \text {, Odebrecht pagó } 6 \\
\text { millones de dólares a dos familiares de un funcionario gubernamental } \\
\text { de alto nivel, a través de fondos no registrados a empresas offshore. }\end{array}$ & 175 millones \\
\hline Argentina & 35.0 & $\begin{array}{l}\text { Entre } 2007 \text { y 2014, Odebrecht realizó pagos corruptos a intermediarios, } \\
\text { en el entendimiento de que estos pagos serían pasados, en parte, a } \\
\text { funcionarios gubernamentales en Argentina. }\end{array}$ & ones \\
\hline Ecuador & 33.5 & $\begin{array}{l}\text { Entre 2007 y 2008, Odebrecht experimentó una serie de problemas } \\
\text { relacionados con un contrato de construcción, y acordó con un } \\
\text { funcionario del gobierno ecuatoriano realizar pagos para resolver los } \\
\text { problemas. Odebrecht luego entregó estos pagos en efectivo al } \\
\text { funcionario del gobierno. }\end{array}$ & 116 millones \\
\hline Perú & 29.0 & $\begin{array}{l}\text { Entre } 2005 \text { y 2014, Odebrecht realizó pagos corruptos a funcionarios } \\
\text { del gobierno en Perú para asegurar contratos de obras públicas. }\end{array}$ & 143 millones \\
\hline Guatemala & 18.0 & $\begin{array}{l}\text { Entre } 2013 \text { y 2015, Odebrecht realizó pagos globales a funcionarios del } \\
\text { gobierno en Guatemala para asegurar contratos de obras públicas }\end{array}$ & 34 millones \\
\hline Colombia & 11.0 & $\begin{array}{l}\text { Entre } 2009 \text { y } 2014 \text {, Odebrecht realizó pagos corruptos en Colombia } \\
\text { para asegurar contratos de obras públicas }\end{array}$ & 50 millones \\
\hline México & 10.5 & $\begin{array}{l}\text { Entre } 2010 \text { y } 2014 \text {, Odebrecht realizó pagos corruptos a funcionarios } \\
\text { del gobierno en México, con el fin de cerrar contratos de obras públicas. } \\
\text { En octubre de } 2013 \text {, Odebrecht acordó el pago de } 6 \text { millones de dólares } \\
\text { a un funcionario de alto nivel de una empresa estatal a cambio de la } \\
\text { obtención del proyecto. }\end{array}$ & 39 millones \\
\hline
\end{tabular}

Fuente: Departamento de Justicia de Estados Unidos (2016a). 
públicos para proyectos de la constructora. Para llevar a cabo los sobornos creó una Caja B en el año 2010 con la finalidad de camuflar las irregularidades en la que estaba incurriendo.

El soborno puede ser activo cuando el delito es cometido por la persona que promete o da el soborno; o puede ser pasivo cuando es cometido por el servidor o persona que recibe el soborno. El soborno puede iniciarse a instancias tanto del funcionario o representante público, como del representante del sector privado. Verdaderamente, es un ciclo viciado que tiene un efecto en cadena y en cascada. Es decir, entre pares y hacia abajo a los empleados de menor rango, dándole a cada uno lo suyo dentro del circuito (Álvarez Severiche, 2017).

Como puede observarse en el amplio periodo de actuación de la constructora, es preciso tomar tiempo para diseñar una estructura para un esquema de sobornos en el que todos los implicados se encuentren en sintonía y no haya mayores inconvenientes en el sistema de sobornos. Esta estructura se creó con la denominación División de Operaciones Estructuradas. Tiene su importancia en la efectividad que mostró durante al menos una década de sobornos. Siendo este un caso tan complejo, se tiene que la estructura logró incorporar a funcionarios públicos de diferentes niveles, además de presidentes, empresarios, así como otras empresas, quienes lograron acuerdos fructíferos y un flujo constante de sobornos gracias a dicha división. De esta manera se constituyó una gran red o cártel por el cual pasó una serie de contratos públicos empañados por acciones de soborno.

\section{Nivel de gravedad}

Tomando en cuenta todo el contexto político desarrollado en el apartado anterior, se tiene, según los criterios desarrollados por Arjona (2002), que el caso Odebrecht responde a un alto nivel de gravedad del acto de corrupción. Esto se evidencia en que el caso ha representado el pago de multa más alto de la historia, una red de corrupción largamente prolongada, y de alcance regional.

Se trata de un estado sistemático de corrupción que alcanzó no solamente a los diferentes gobernantes de la región, sino en una variedad de cargos públicos de todos los niveles, no solo políticos, sino judiciales. Las operaciones en cada uno de los Estados afectados mantuvieron la misma dinámica, por lo que es posible señalar que se implementó una estructura que creó las condiciones de corrupción política persistentes. Por su parte, la acción del soborno lleva implícita una decisión de tipo ético respecto a la forma de proceder ante el mismo, y estas se manifiestan según la robustez ética del funcionario y, por lo tanto, de las instituciones públicas. 
Cuando se evalúan los costos que representa para las políticas latinoamericanas la aceptación de los sobornos de la empresa Odebrecht, se tiene que efectivamente no ha habido una mejora en cuanto al desenvolvimiento social, la economía, ni mucho menos la estabilidad política de los países afectados.

\section{Actores que intervienen en el acto de corrupción y el grado de implicación}

Como se ha dicho, el caso Odebrecht ha salpicado no solo a una línea de gobernantes de la región, sino que ha tenido influencia sobre diversos niveles de cargos públicos como lo son alcaldes, gobernadores, jueces, entre otros.

El mayor grado de implicación lo tuvieron Venezuela y Colombia, según el índice de percepción de corrupción. En ambos países se encuentran implicadas las principales figuras políticas; en el caso de Colombia se encuentran señalados el ex presidente Álvaro Uribe, el ex viceministro de Transporte Gabriel García Morales y el senador Otto Nicolás Bula.

Las relaciones entre los ex mandatarios de Colombia y Brasil tuvieron muchos roces debido a que durante la presidencia de Uribe hubo mucha confrontación con el fallecido presidente Hugo Chávez que sí era apoyado por Lula da Silva. Esto comprueba que aunque las alianzas políticas no fueron perfectas los casos de corrupción se abrieron paso sin muchas complicaciones.

En el caso de Venezuela, se encuentran implicadas diferentes figuras de la cúpula política como lo es el actual presidente de Venezuela Nicolás Maduro como funcionario principal.

Los sobornos de Odebrecht se utilizaron para el financiamiento de las desorbitadas campańas electorales de Chávez, por lo que las declaraciones que provienen del caso expresan pagos de hasta 11 millones de dólares. Mientras que para la campaña electoral de Maduro se recibieron unos 35 millones (2013).

\section{Ámbito en el cual sucede el hecho de corrupción}

El caso de corrupción Odebrecht alcanzó a unas doce naciones, siendo las más perjudicadas Brasil, Argentina, Colombia, República Dominicana, Guatemala, Ecuador, Panamá, México, Perú y Venezuela. En los casos de Venezuela, Ecuador y Argentina sucedieron expresamente en el contexto electoral que permitió la reelección de los presidentes o, en todo caso, la continuidad de aliados políticos.

\section{El daño impuesto en el sistema político}

El caso más emblemático que se deriva de la corrupción directamente relacionada con Odebrecht es el venezolano. La incidencia de los sobornos de la constructora han 
GRÁFICA 3. Crecimiento de América Latina, 2017

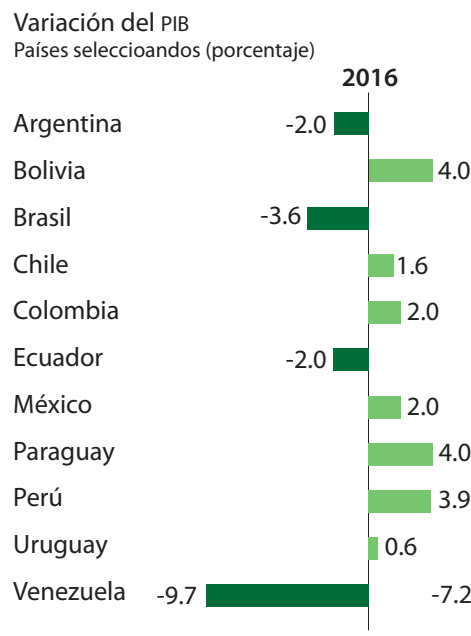

Evolución del PIB

América Latina y el Caribe (porcentaje)

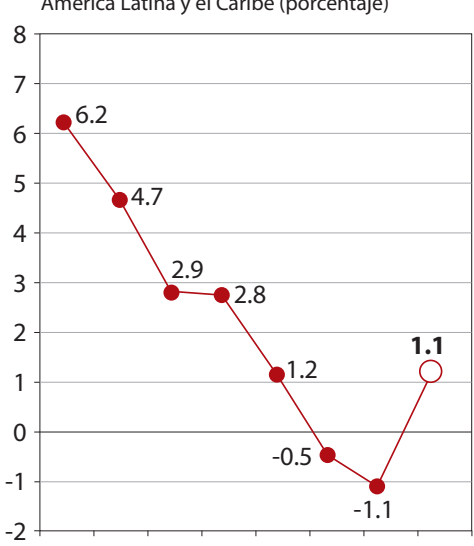

2010201120122013201420152016 2017*

Fuente: CEPAL (2017). *Nótese la gran diferencia económica entre Venezuela y los otros países latinoamericanos.

beneficiado la permanencia en el poder político del régimen chavista-madurista que ha afectado negativamente al país y que ha provocado una crisis humanitaria aguda.

Aunque la causa directa de la situación de Venezuela no recae directamente en Odebrecht, se tiene que el sistema de sobornos permitió otorgar los medios económicos para que se perpetúe el gobierno que lo rige desde hace casi veinte ańos.

Hoy en día Venezuela es el país con más retraso de la región, como se presenta en la gráfica 3 .

Adicionalmente, Venezuela sufre paralelismo en varias de sus instituciones y un sistema electoral que es rechazado mundialmente por celebrar elecciones fraudulentas. Estos puntos se desarrollarán en los próximos apartados.

\section{Sectores Involucrados}

Los sectores involucrados están representados en el privado por la constructora Odebrecht, y el público en cada uno de los países afectados.

\section{Centralización y descentralización de los actos de corrupción}

Debido a que la constructora Odebrecht estableció un modo operativo sistemático de sobornos, su influencia no solo alcanzó a la cúpula política de los 
países afectados. En el caso de Colombia tuvo una fuerte influencia en la región Río de Oro-Aguachica-Gamarra en proyectos que fueron aprobados por el Congreso colombiano, además de las autoridades locales. La facilidad de la estructura corrupta trazada por la constructora le permitió colarse en diferentes niveles de poder.

\section{Influencia sobre la legitimidad democrática en la región}

El escándalo de corrupción en la región latinoamericana ha descubierto una red de sobornos que evidencia un esquema de corrupción que ha sido erigido sin atender a ninguno de los valores éticos, y sin reparo de perjuicios generando una percepción de que en la región persiste una situación que se encuentra inscrita en su idiosincrasia. Más allá de las imposiciones y sanciones legales, el hecho ha ocasionado el deterioro del patrimonio relacionado con las infraestructuras en las que intervenía la empresa, y que representaba un avance para los países afectados.

Pero también la pérdida de la legitimidad en América Latina por influencia de Odebrecht en la persistencia de líderes de corte socialista que acabaron por afectar el avance de la región se evidencia en las crisis políticas que se generaron a partir de este hecho. El caso ha llamado la atención internacional por la compleja red de sobornos que la constructora logró tejer durante al menos una década.

No solo la persistencia, sino el afincamiento de la corrupción política en América Latina configuró una alianza política entre líderes populares de izquierda que tuvo especial epicentro en Brasil gracias a la popularidad de Lula da Silva, y del fallecido presidente Hugo Chávez de Venezuela. De esta manera, persiste una explosión de casos reales de corrupción que afectan de manera negativa los sistemas democráticos de los países afectados, sino que también persiste la creencia de que la corrupción es una especie de enfermedad propia de la región.

Esta percepción incide desfavorablemente en la ralentización de los profundos problemas sociales que caracterizan a América Latina. Esta situación crea constante malestar en la población

A excepción de unos pocos países, la corrupción es endémica en toda América Latina. A pesar de la retórica de los dirigentes políticos de todas las tendencias ideológicas, afianzada por tratados regionales, planes nacionales y miles de campañas, la región ha hecho escasos progresos para acabar, o incluso modestamente frenar, las prácticas de corrupción en los últimos veinte años... La corrupción aparece como un elemento permanente y generalizado en casi todos los países latinoamericanos (Llorente \& Cuenta, 2016). 
La legitimación de la democracia se ha visto afectada en tanto que los Estados latinoamericanos no han logrado avanzar en medidas efectivas contra la corrupción política. En algunos casos la corrupción se ha visto tan expandida dentro del sistema político que combatir sus efectos ha resultado muy difícil, por no decir imposible.

Siendo que en los casos más graves relacionados con Odebrecht el hecho de corrupción se estableció en el contexto electoral, la fragilidad democrática ha sido una de las consecuencias más visibles de este caso, y se evidencia en la perpetuación en el poder de ciertos líderes políticos como en el caso venezolano y colombiano, y en la constante apertura de expedientes judiciales como en el caso brasileño, argentino, peruano, entre otros.

Vale la pena reflexionar entonces acerca del comportamiento individual de los países afectados y las medidas que tomen para subsanar la situación de corrupción que ha afectado a toda la región, sobre todo cuando existen las bases internacionales para la cooperación y la superación de este tipo de fenómeno político. En este sentido, cabe preguntar cuáles son las medidas que pueden tomarse a nivel regional sobre los beneficios que se obtuvieron a través de actos delictivos de corrupción, cuáles son las sanciones que se aplican al caso y, sobre todo, cuáles son las acciones de saneamiento de las instituciones que reorientarán las políticas nacionales a un Estado robusto y ético de la democracia.

\section{Evidencia en la inestabilidad política}

La inestabilidad política más evidente tiene tres expresiones claramente identificadas: Brasil, Venezuela, Argentina. En Brasil se evidencia en la destitución de la presidenta Dilma Rousseff y en las constantes manifestaciones sociales que siguieron como consecuencia a este hecho. En Venezuela se evidencia a partir de los efectos de la dictadura de Nicolás Maduro que ha provocado una crisis humanitaria, además de largos periodos de movilizaciones sociales en los últimos cuatro años.

El gobierno venezolano sufre hoy día un rechazo generalizado, viéndose sancionado por diferentes esferas políticas internacionales incluyendo la Comisión Europea, Estados Unidos, entre otros.

La situación de corrupción política en Venezuela puede ser catalogada como sistémica debido a la crisis política actual institucional que presenta. En este sentido se evidencia que las políticas populistas y totalitarias primero del chavismo, y ahora del madurismo han provocado un desgaste significativo del poder público en el cual existe la duplicidad de las instituciones. La corrupción política se evidencia además en el desfalco económico realizado por el gobierno a través del sistema del control cambiario y del dominio de la petrolera estatal Petróleos de Venezuela (PDVSA). 
Siguiendo la línea de los autores citados, en los casos de corrupción política y sistemática se presenta la mayor dificultad de que es más difícil imponer las sanciones adecuadas, en primer lugar por la misma contaminación corruptiva de las instituciones, y en segundo lugar porque el hecho corruptivo se convierte en el modus operandi por el cual funciona el gobierno en todas sus escalas.

En cuanto al caso de Argentina, se expresa en la influencia que todavía ejerce la ex presidenta Cristina Kirchner quien, a pesar de llevar expedientes judiciales, aún persiste en la esfera política del país. La presidenta Cristina Kirchner ha dejado una administración en la cual Argentina sufre un grave atraso económico y social, así como una sociedad altamente dividida. Esto ha perjudicado el avance del presidente Macri en la recuperación del país.

\section{Influencia en la debilidad de las instituciones}

Los países que presentan debilidad en sus instituciones crean las condiciones ideales para que se expanda la corrupción de manera sistemática. Como se ha demostrado, aunque Brasil es el epicentro del caso Odebrecht, la fortaleza de su sistema judicial ha sido esencial para que este país retome el camino a la normalidad. Esto se evidencia en que Brasil ha bajado en el índice de percepción de corrupción teniendo cierta recuperación.

Sin embargo, en el caso de Venezuela, el más grave del grupo estudiado, hoy en día este país sufre de paralelismo en dos de sus poderes públicos: la Asamblea Nacional y el Tribunal Supremo de Justicia.

Tanto la debilidad democrática como de las instituciones se expresan en la disolución de la legítima Asamblea Nacional electa popularmente en 2015. En su lugar, el gobierno venezolano celebró unas elecciones fraudulentas que sustituyó la asamblea con funcionarios exclusivamente alineados con el gobierno.

Por otra parte, la conformación de un nuevo Tribunal Supremo que funciona en el exterior y que es coordinado por sectores de la oposición demuestra la mayor fragilidad de instituciones de la región en los países estudiados.

\section{CONCLUSIONES}

El caso Odebrecht ha mostrado que en el contexto latinoamericano la corrupción es un problema de largo alcance en cuanto a magnitud del hecho expresado en la cantidad de países afectados, así como a la variedad de figuras políticas de diferentes rangos y tendencias políticas. Se trata de un hecho que ha conmocionado a la región y que ha tenido consecuencias bastante graves en ciertos casos como el de Brasil, Perú, Argentina y Venezuela. 
En este trabajo se ha realizado una revisión teórica profunda que permite el abordaje de la corrupción generada por Odebrecht en América Latina, desde el punto de vista de la corrupción política. Se tiene que efectivamente en los países analizados se ha generado una debilidad de la legitimación de las instituciones, provocando inestabilidad política y retraso en el desarrollo regional respecto al resto del mundo. A partir de las perspectivas metodológicas, en el caso de esta investigación nos referimos a la corrupción política en América Latina según lo que se ha estudiado, la cual se caracteriza por los sobornos, extorsiones, fraudes y malversaciones relacionadas con el caso Odebrecht.

Según el análisis realizado, el caso Odebrecht en América Latina ha alcanzado un alto nivel de gravedad debido a su actuación sistemática de soborno. De manera que la diversidad de funcionarios públicos afectada es considerable. Al mismo tiempo, se trata de un caso que involucra en un mismo escenario lo público con la empresa privada, por lo que se desprende del mito que la corrupción latinoamericana se presenta exclusivamente en el plano político.

Además, debido a la sistematización de los sobornos, Odebrecht se abrió paso a figuras políticas de diferentes tendencias, y aunque Brasil es considerado el epicentro de esta red corruptiva, no todas las relaciones de este país con sus vecinos fueron del todo estrechas (casos de Colombia y Ecuador). Sin embargo, la constructora Odebrecht ha sabido adaptarse a cada escenario y ha sabido trazar su estrategia de sobornos de manera efectiva en cada caso.

Aun así, es posible confirmar que en algunos casos las alianzas políticas como las conformadas desde Brasil con Perú y Venezuela fueron bastante productivas para la constructora. En última instancia, la constructora Odebrecht supo leer las oportunidades en procesos electorales, como el del presidente Maduro de Venezuela o el ex presidente Kuczynski de Perú.

Es indudable que el caso Odebrecht ha salpicado a la región, causando lo que algunos analistas llaman "terremoto político", debido a su prolongado trayecto y alcance. En el complicado entramado internacional que implica el caso se incluye una lista importante de figuras de la esfera política latinoamericana.

En este sentido, el caso de corrupción política que involucra a la empresa Odebrecht es emblemático, y sus consecuencias aún están por medirse en algunos de los países afectados. Sin embargo, se evidencian claramente sus efectos en tres escenarios específicos: Brasil, Perú y Venezuela, los cuales están sufriendo una inestabilidad política que debilita la legitimidad de sus instituciones y su crecimiento económico.

Aunque la investigación ha arrojado que no todos los países latinoamericanos sufren el mismo índice de corrupción, la percepción de la región es que la corrup- 
ción política sigue siendo un mal endémico regional, que se percibe como generalizado y persistente.

En este sentido, los países afectados fallan al carecer de medidas de efectivas que les haga retroceder en los hechos de corrupción política. Se ha creado una cultura de la corrupción, como en el caso de Colombia y Venezuela, en la que no solo la cúpula política se ha visto afectada, sino que se trata del sistema público en toda su dimensión. Esta situación ha hecho más difícil combatir este problema.

Países como Venezuela, que permiten el debilitamiento de las instituciones, presentan una debilidad crítica de su sistema político afectando su crecimiento económico y generando una crisis humanitaria como la que experimenta actualmente. La corrupción que sufre este país ha provocado el paralelismo de sus instituciones y el rechazo mundial, siendo el caso más grave de todos los analizados.

De esta manera, esta investigación ha intentado contribuir para entender cómo la red de corrupción impulsada por Odebrecht ha tenido diferentes efectos en los países afectados, pero también como un análisis que permite observar las consecuencias de este caso.

La disposición de los países latinoamericanos por buscar el saneamiento y la renovación de las instituciones será el primer paso para rescatar los espacios democráticos y avanzar en materia de vencimiento de la corrupción política. Unas instituciones fortalecidas significan el freno para el efecto contaminante que tiene la corrupción en los diferentes niveles del escenario público.

Por último, esta investigación representa una base para avanzar en temas que involucran a los países concretos afectados, así como para realizar análisis comparativos como propuestas de investigaciones futuras. En cuanto a los retos que ha afrontado la investigación se tiene que alguno de los hechos son bastante recientes, por lo que su abordaje se hace desde la perspectiva actual.

Debido a la magnitud del caso, los escándalos de corrupción se seguirán destapando, por lo que se entiende que el análisis aún está abierto para la futura profundización. Gミ

\section{REFERENCIAS}

Actis, E. (2012), "Las desavenencias en el vínculo económico entre Argentina y Brasil: El turno de las inversiones (2010-2012)", Revista Integración y Cooperación Internacional, 13, pp. 18-29.

Actis, E. (2015), "Estrategias de desarrollo e internacionalización de capitales: Pasado y presente del caso brasileño", Tempo do Mundo, 1(2), pp. 45-68. 
Álvarez Severiche, N.A. (2017), La acción popular en la restitución de los daños causados a la nación caso Odebrecht, Bogotá, Universidad Santo Tomás de Bogotá.

Arcos, X., P. Cardinale, L.M. Castilla, Q. Franco, R. García, F. Jaramillo, L.C. Jemio, O. Manzano, P. Rojas, G. Ríos, M.T. Szauer, E. Urquillas, N. Villoria y M. Zambrano (2002), Temas criticos para América Latina, Quito, Banco de Desarrollo de América Latina (CAF), disponible en: http://scioteca.caf.com/handle/123456789/671 [fecha de consulta: 11 de noviembre de 2019].

Arjona Trujillo, A.M. (2002), "La corrupción política: Una revisión de la literatura”, documento de trabajo 02-14, Universidad Carlos III de Madrid.

Arroba, F.O. (2010), "Relaciones comerciales Ecuador-Brasil: Un tablero sin alfiles", Comentario Internacional, 10, pp. 125-137.

Avritzer, L. y F. Filgueira (2011), Corrupção e controles democráticos no Brasil, Instituto de Pesquisa Econômica Aplicada, Brasilia, CEPAL.

Caracol Radio (2017), "Más de 100.000 investigaciones adelanta la Fiscalía por casos de corrupción”, 5 de abril.

Casar, M.A. (2015), México: Anatomía de la corrupción, Ciudad de México, Cide/IMCo, disponible en: https://imco.org.mx/wp-content/uploads/2015/05/2015_Libro_completo_Anatomia_corrupcion.pdf.

CAF (Banco de Desarrollo de América Latina) (2012), Focus América Latina, 2, Caracas, CAF, disponible en: http://scioteca.caf.com/handle/123456789/160 [fecha de consulta: 10 de noviembre de 2020].

CAF (Banco de Desarrollo de América Latina) (2015), Focus América Latina, 7, septiembre, Buenos Aires, CAF, disponible en: http://scioteca.caf.com/handle/123456789/797 [fecha de consulta: 10 de noviembre de 2020].

Cedeño-Brea, E. (2017), “Odebrecht, bancos dominicanos y clientes Pep”, Blog LexMercatoria, 8 de enero, disponible en: https://enmanuelcedenobrea.com/2017/01/08/odebrecht-bancos-dominicanos-y-clientes-pep/ [fecha de consulta: 23 de mayo de 2020].

Cevallos Guerrero, N. (2012), "La influencia político-comercial en las relaciones entre Ecuador y Brasil en los casos Petrobras y Odebrecht", tesis de licenciatura, Universidad Internacional del Ecuador.

Cousinou, G.M. (2006), "La corrupción política: Nuevas aportaciones para un viejo debate", Revista de Estudios Políticos, 133, pp. 197-221.

CNN Español (2016), "Dilma Rousseff, la presidenta que hizo historia en Brasil 2 veces en menos de 6 años", 12 de mayo.

Correa, M.V. (2017), "Las megaobras de Odebrecht, tras 24 años en Colombia”, El Colombiano, 8 de enero.

Couto, C.G. (2011), "O governo Lula e as perspectivas de Dilma Rousseff”, Iberoamerica- 
na: América Latina-España-Portugal, 11(41), pp. 159-163, DoI: https://doi.org/10.18441/ ibam.11.2011.41.159-163.

Covarrubias González, I. (2005), "La globalización de la corrupción: Un efecto perverso de las transformaciones recientes en la política y la democracia”, Bajo el Volcán, 5(9), pp. 13-39.

De la Cuadra, F.M. (2014), "Expansión de las fronteras internas de América Latina: El papel de Brasil en la integración regional”, Polis, 13(39), pp. 7-20.

De Melo, C.A.V. (2015), "Elementos constitutivos e dinâmicos da corrupção: Um exercício conceitual", Arius, 17(2), pp. 51-73.

Departamento de Justicia de Estados Unidos (2016a), documento del 21 de diciembre.

Departamento de Justicia de Estados Unidos (2016b), “Odebrecht and Braskem Plead Guilty and Agree to Pay at Least \$3.5 Billion in Global Criminal Penalties to Resolve Largest Foreign Bribery Case in History", julio de 2017, Washington, D.C., Departamento de Justicia de Estados Unidos, disponible en: https:/www.justice.gov/usao-edny/pr/odebrecht-and-braskem-plead-guilty-and-agree-pay-least-35-billion-global-criminal [fecha de consulta: 20 de octubre de 2020].

Deshazo, P. (2007), "Esfuerzos contra la corrupción en América Latina: Lecciones aprendidas", documentos de Política sobre las Américas, XVIII, estudio 2, csis.

Dinatal, M. (2016), "Observó la oEA fallas en la lucha contra la corrupción”, La Nación, 18 de octubre.

Díaz, E.M. y D.P. Raimán (2017), "Clientelismo y corrupción en contextos de baja estatalidad, una relación mutualista”, Revista de Sociologia e Política, 25(64), pp. 73-98.

Diego Bautista, O. (2006), "La ética y la corrupción en la política y la administración pública”, tesis de maestría, Universidad Internacional de Andalucía.

Europa Press (2015), "Una comisión parlamentaria se constituye para investigar al Banco de Desarrollo de Brasil", 6 de agosto.

El Heraldo (2017), "Fiscales de Argentina y Brasil denuncian obstáculo en investigación en caso Odebrecht", Sección Mundo, 1 de agosto.

El Mundo (2017), "Odebrecht se declaró culpable de una información criminal de un recuento presentada hoy por la Sección de Fraudes de la División de lo Penal y la Oficina del Fiscal Federal en el Tribunal de Distrito de Nueva York, acusando a la compañía de conspiración para violar las disposiciones contra el soborno de la Ley de Prácticas Corruptas en el Extranjero (FCPA)", 14 de julio.

El Tiempo (2017), "Estos son los políticos implicados en el caso Odebrecht en Brasil", 12 de abril.

Flores, L.P. (2015), “Caso Petrobras: Análisis y reflexión del fraude corporativo”, Revista Lidera, 10, pp. 85-87. 
Galli, C.M. (1996), "La corrupción como pecado social”, en G. Farrel-D. García DelgadoF. Forni, Tiempo de cambios, Buenos Aires, Ediciones San Pablo.

Gonçalves, A.F. (2003), "La cultura y el mercado en el contexto transnacional: Una etnografía de la tecnología empresarial Odebrecht”, tesis doctoral, Universidad Estatal de Campinas.

González Amuchástegui, J. (1999), "Corrupción, democracia y responsabilidad política”, Isonomía: Revista de Teoría y Filosofía del Derecho, 10, pp. 7-24.

Hernaíz, C.E., M. Phélan y J. Camacho (2014), "Sobre las mediciones de corrupción y su relación con el desarrollo y el bienestar en América Latina”, Paradigmas, 6(2), pp. 131-177.

Herrera Niño, S.F. (2017), "Eficiencia, eficacia y transparencia: Pilares de la gestión pública en Colombia", tesis de licenciatura, Universidad Militar Nueva Granada.

Herrero, M. (2003), "Legitimidad política y participación", Anuario Filosófico, XXXVI(1), pp. 111-134.

Iturralde, G. (2014), "Análisis del intercambio comercial de bienes entre Ecuador y Brasil durante el periodo 2007-2012“, tesis de licenciatura, Pontificia Universidad Católica del Ecuador.

Heidenheimer, A.J., M. Johnston y V.T. Le Vine (eds.) (1989), Political Corruption: A Handbook, New Brunswick, Transaction Publishers.

Infobae (2017), "El nuevo ranking de corrupción en América Latina expone la percepción global: Venezuela sigue en la peor posición“, 25 de enero, disponible en: http://www. infobae.com/america/america-latina/2017/01/25/el-nuevo-ranking-de-corrupcionen-america-latina-expone-la-percepcion-global-venezuela-sigue-en-la-peor-posicion/ [fecha de consulta: julio de 2017].

La Vanguardia (2017), "Lula da Silva, condenado a nueve ańos y medio de prisión por corrupción", 12 de julio.

La Nación (2016), "Odebrecht: El mapa de los negocios millonarios en la Argentina”, 23 de diciembre.

Laporta, F.J. y P.A. Ibáńez (1997), La corrupción política, Madrid, Alianza.

Leahy, L. (2017), “Caso Odebrecht 'dibuja' la corrupción en Brasil”, El Financiero, 25 de abril.

Lissardy, G. (2013), “¿Por qué crecen cada vez más las protestas en Brasil?” 21 de junio, BвC. López, N.R. (2011), "La eficiencia y su importancia en el sector público", eXtoikos, 1, pp. $38-47$.

Llorente \& Cuenca (2016), "La corrupción, el talón de Aquiles de las democracias latinoamericanas", informe especial, Madrid, Desarrollando Ideas: Centro de Liderazgo a través del Conocimiento de Llorente \& Cuenca, disponible en: https://ideas.llorenteycuenca. 
com/wp-content/uploads/sites/5/2016/09/160912_DI_informe_Corrupcion_LatAm_ESP.pdf [fecha de consulta: 2 de abril de 2019] .

Machado, Aads (2008), "A Dilemma for the Brazilian Regional Leadership: The Case of Odebrecht/O caso Odebrecht e o dilema da lideranca Regional Brasileira", Meridiano 47(100), pp. 32-37.

Máiz, R. (2005), "El clientelismo de partido y la corrupción política”, en J. Montabes (coord.), Instituciones y procesos politicos: Libro homenaje a José Cazorla, Madrid, Centro de Investigaciones Sociológicas.

Mansilla, H.C.F. (2000), "Algunas insuficiencias de la democracia contemporánea: Una crítica de las teorías de la transición latinoamericana", Revista de Estudios Políticos, 108, pp. 77-102.

Menéndez Quevedo, X. (2017), El cuento de hadas del buen gobierno corporativo: El pecado capital de Odebrecht, Panamá, Instituto de Gobierno Corporativo de Panamá.

Mohabbat, M. (2004), "Political and Administrative Corruption: Concepts, Comparative Experiences and Bangladesh Case", preparado para Transparency International, capítulo Bangladesh.

Mudde, C. (2010), “The Populist Radical Right: A Pathological Normalcy”, West European Politics, 33(6), pp. 1167-1186.

Naím, M. (2017), “Corrupción: ¿Héroes o leyes?”, El País, 18 de noviembre.

Neely Ivanovic, C. (2010), Crónica de la corrupción a la chilena, Santiago de Chile, Editorial Cuarto Propio.

OEA (Organización de los Estados Americanos) (1996), Convención Interamericana contra La Corrupción, 29 de marzo, disponible en: http://www.oas.org/es/sla/ddi/docs/tratados_multilaterales_interamericanos_B-58_contra_Corrupcion.pdf [fecha de consulta: septiembre de 2017].

OEA (Organización de los Estados Americanos) (1998), Convención Interamericana contra la Corrupción, resolución núm. 489-98 del 1 de noviembre, disponible en: http://www. oas.org/juridico/spanish/dna_res5.pdf [fecha de consulta: septiembre de 2017].

Patiño Aristizábal, L.G. (2007), "El neopopulismo en el contexto de la democracia latinoamericana", Revista Facultad de Derecho y Ciencias Politicas, 37(106), pp. 239-261.

Parker, N., G. Berthin, R. De Michelle y Y. Mizrahi (2004), La corrupción en América Latina: Estudio analítico basado en una revisión bibliográfica y entrevistas, Virginia, USAID/ Casals \& Associates.

Perfil (2016), "Las ocho obras de Odebrecht en Argentina que investiga la Justicia", Sección Política, 22 de diciembre.

Pimentel Jr, J. (2015), "Impeachment, oposición y autoritarismo: El perfil y demanda de los manifestantes en São Paulo", Em Debate, 7(2), pp. 15-22. 
Quiroz, A.W. (2014), Historia de la corrupción en el Perú, Lima, Instituto de Estudios Peruanos.

Rózga-Luter, R.E. y C. Hernández-Diego (2010), “Los estudios regionales contemporáneos: Legados, perspectivas y desafíos en el marco de la geografía cultural", Economía, Sociedad y Territorio, 10(34), pp. 583-623.

Rodríguez Kauth, A. (2011), "Corrupción e impunidad: Dos estilos de cultura política latinoamericana”, Investigación y Desarrollo, 8(3).

Rodrigues, A. y J.S. Gomes (2006), "Controle gerencial em empresas internacionalizadas: O caso da construtora Noberto Odebrecht S.A.”, Revista Contemporânea de Contabilidade, 3(5), pp. 75-96.

Rabotnikof, N. (1999), "Corrupción política: definiciones técnicas y sentidos sedimentados", Isonomia: Revista de Teoría y Filosofía del Derecho, 10, pp. 25-39, disponible en: http:// www.cervantesvirtual.com/research/corrupcin-poltica-definiciones-tcnicas-y-sentidossedimentados-0/006aafc0-82b2-11df-acc7-002185ce6064.pdf [fecha de consulta: 15 de julio de 2019].

Riquelme, R. (2017), “América Latina vive sumida en la corrupción”, El Economista, 28 de enero.

RPP Noticias (2017a), "La relación entre Lula da Silva y el expresidente Ollanta Humala", 11 de abril.

RPP Noticias (2017b), “6 claves para entender la operación 'Lava Jato”, 19 de mayo. Segré, G. (2017), “Corrupción, Odebrecht y la rama argentina”, El Clarín, 7 de mayo.

Stuckert Filho, R. (2016), "PT cierra ciclo de 13 años en el poder con la bandera de la ética al contado", Estado de Minas, Sección Política, 21 de agosto.

Terán, T. y M. Alejandro (2009), "Factores explicativos y dimensiones de la estabilidad política: un estudio mundial”, tesis doctoral, Flacso-México.

Transparencia Internacional (2017), "Recursos: CPI 2016 Full Data Set With Regional Tables" julio, disponible en: web: https://www.transparency.org/news/feature/corruption_perceptions_index_2016 [fecha de consulta: 5 de enero de 2020].

Tible, J. (2015), “¿Fin de fiesta en Brasil?” Nueva Sociedad, 259, pp. 4-14.

Villoria Mendieta, M. (2006), “PPor qué desconfiamos de los políticos? Una teoría sobre la corrupción de la política”, Revista del CLAD Reforma y Democracia, 34, pp. 1-21.

Westin, R. (2017), A queda de Dilma, São Paulo, Universo dos Livros Editora.

Zéphyr, D. (2008), "Corruption and Its Impact on Latin American Democratic Stability", en M.A. Seligson (ed.), Challenges to Democracy in Latin America and the Caribbean: Evidence from the AmericasBarometer, 2006-2007, Vanderbilt University-LAPOP, pp. 251-276. 
Song Yuhui tiene el título de máster por la Facultad de Lenguas Extranjeras, Universidad de Finanzas y Economía de Guizhou (GUfE), China. Sus principales líneas de investigación son la lingüística española, así como la política, la cultura y las relaciones internacionales de los países españoles y portugueses. Desde septiembre de 2018 trabaja como profesor de chino en el extranjero, en el Instituto Confucio de la Universidad de Cabo Verde (UNI-CV). 